medRxiv preprint doi: https://doi.org/10.1101/2021.05.23.21257669; this version posted May 25,2021 . The copyright holder for this preprint (which was not certified by peer review) is the author/funder, who has granted medRxiv a license to display the preprint in perpetuity.

It is made available under a CC-BY-NC-ND 4.0 International license .

\title{
Exploiting Molecular Basis of Age and Gender Differences in Outcomes of SARS-CoV-2 Infections.
}

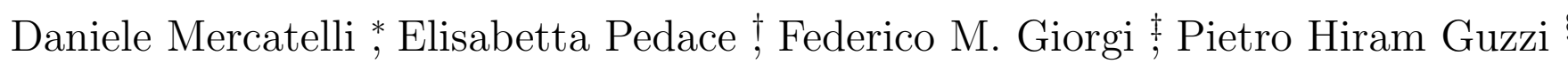
May 23, 2021

\footnotetext{
*University of Bologna

${ }^{\dagger}$ ASP Catanzaro

University of Bologna, Department of Pharmacy and Biotechnology.

$\S$ University of Catanzaro, Department of Medical and Surgical Sciences
} 


\begin{abstract}
Motivation: Severe acute respiratory syndrome coronavirus 2 (SARS-CoV-2) infection (coronavirus disease, 2019; COVID-19) is associated with adverse outcomes in patients. It has been observed that lethality seems to be related to the age of patients. Moreover, it has been demonstrated that ageing causes some modifications at a molecular level. Objective: The study aims to shed out light on a possible link between the increased COVID-19 lethality and the molecular changes that occur in elderly people. Methods: We considered public datasets on ageing-related genes and their expression at tissue level. We selected interactors that are known to be related to ageing process. Then, we performed a network-based analysis to identify interactors significantly related to both SARS-CoV-2 and ageing. Finally, we investigated changes on the expression level of coding genes at tissue, gender and age level.

Results We observed a significant intersection between some SARS$\mathrm{CoV}-2$ interactors and ageing-related genes suggesting that those genes are particularly affected by COVID-19 infection. Our analysis evidenced that virus infection particularly affects ageing molecular mechanisms centred around proteins EEF2, NPM1, HMGA1, HMGA2, APEX1, CHEK1, PRKDC, and GPX4. We found that HMGA1, and NPM1 have a different expression in lung of males, while HMGA1, APEX1, CHEK1, EEF2, and NPM1 present changes in expression in males due to aging effects.

Conclusion Our study generated a mechanistic framework to explaining the correlation between COVID-19 incidence in elderly patients and molecular mechanisms of ageing. This will provide testable hypotheses for future investigation and pharmacological solutions tailored on specific age ranges.

Keywords: Data Science, SARS-CoV-2, COVID-19, Ageing Genes, Interactomes

Contact: Pietro Hiram Guzzi, Department of Surgical and Medical Sciences, University of Catanzaro hguzzi@unicz.ithguzzi@unicz.it
\end{abstract}

\title{
1 Introduction
}

At the end of 2019 in Wuhan (China), medical facilities reported acute pneumonia cases with an unknown origin. Further analysis revealed that a novel coronavirus, named severe acute respiratory syndrome coronavirus 2 (SARS-CoV-2), was responsible for that disease, subsequently called coronavirus disease 2019 (COVID-19) [1, 2]. The clinical manifestations spanned from asymptomatic infection to severe 
pneumonia and a severe state of inflammation (molecularly characterised by a cytokine storm) leading to a fatal outcome $[3,4,5,6,7,8]$.

Starting from China, the virus spread in almost all other countries globally, causing infections and deaths. On 11th March 2020, the World Health Organisation (WHO) declared SARS-CoV-2 as a pandemic. Current data revealed that the impact of COVID-19 presents certain peculiar aspects in different nations that have been deeply investigated $[9,10]$. Some authors hypothesised that virus mutations were responsible for these differences $[11,12,13,14]$. Nevertheless, many independent studies agreed that the mutations might not have a primary role in explaining these differences $[15,16,17]$.

Despite the lack of the individuation of the causes, there was a substantial agreement on the fact that the variation of the observed case fatality rate (CFR), i.e. the fraction of confirmed cases leading to fatal outcomes, ranging from 0 to $20 \%$ and beyond at country level, needs to be deeply investigated $[18,19,20]$. Among the other differences, we focused on observing that the infection is significantly more lethal in older people $[21,22,23,24,25]$. This consideration has also guided the optimisation of vaccination strategy [26].

Some studies have focused on the possible link between increased mortality rate and some characteristics of older people [27, 28]. In addition, these studies suggested the potential effect of the virus as a trigger activating the decompensation of other chronic conditions [29, 30, 31, 32]. Akbar et al., [33], discussed a possible link between the increased chronic inflammatory status occurring during ageing (termed "inflammaging" $[34,35]$ ), and COVID-19 manifestation that causes the rise of inflammation.

Previous studies have also evidenced that the understanding of modification of molecular mechanism related to the ageing process (i.e. modification of gene expression, modulation of regulatory mechanisms) may reveal important insights about ageing [36]. Many studies contributed to identifying such ageing-related diseases despite the lack of having experimental data $[37,38,39,35,40]$. Computational predictions have also been made in $[36,41]$ giving both candidate genes and networks $[42,43]$.

Consequently, the study of the intersection between SARS-CoV-2 and ageing-related molecular alterations could augment the understanding of COVID-19, thus improving treatment options [44]. Bhattacharyya et al. presented a first analysis based on some preliminary public data reinforcing the rationale that such a possible link exists $[45]$.

Six functional open reading frames (ORFs) in the SARS-CoV2 genome encodes for the four main structural proteins, the Spike 
(S), Envelope (E), Membrane (M), and the Nucleocapsid (N), and ORF1a/ORF1b, which contain information for the replicase-transcriptase complex formed by 16 non-structural proteins (NSP1-NSP16). The SARS-CoV-2 genome also contains 9 accessory factors from sub-genomic ORFs (Orf3a, 3b, 6, 7a, 7b, 8, 9b, 9c and 10) [46]. We investigated the relationships and interactions between these viral components and age-related factors and observed a significant overlap between SARSCoV-2 and ageing group genes' interactors. Furthermore, we looked at a network-level scenario [43], by considering possible regulatory mechanisms that may be altered $[47,48]$. These observations support previous reports that SARS-CoV-2 also involves a vascular and multiorgan failure in severe COVID-19 [49].

Starting from these considerations, we hypothesised that SARSCoV-2 interacting proteins (and genes) might show an overlap with human ageing-related genes higher than chance. Therefore, the infection may deregulate these mechanisms that can be already impaired in older adults, causing severe outcomes. We downloaded public available interaction data from Guzzi et al. [50] and Gordon et al., [51]. Then we considered the interacting partners that were annotated as ageing genes in MSigDB [52] ad we also considered the expression at tissue and sex levels extracting data from GTeX database [53]. We verified a significant fraction of interacting partners of SARS-CoV-2 involved in ageing. These genes are also expressed in lung and the expression is modulated by age and sex. We also observed that these genes are expressed in adipose tissue (as reported in Supplementary Material). The workflow of the experiment is depicted in Figure 1.

\section{Methods}

SARS-CoV-2 Interaction Map We considered the SARS-CoV2 protein interaction map provided by Gordon et al., [51], and by Guzzi et al., [50]. Both works provided data about 26 of the 29 SARS-CoV-2 proteins behaviour in human cells by identifying the human proteins that physically associated with each of the SARS-CoV-2 proteins using affinity-purification mass spectrometry. They found high-confidence protein-protein interactions between SARS-CoV-2 and human proteins; they also provided data about possible interactions with an associated reliability score. We considered both high and low confidence interactions.

Databases We first defined and labelled genes related to the ageing process as ageing. Then, we considered data provided from the GTEx dataset containing genes positively and negatively correlated 
medRxiv preprint doi: https://doi.org/10.1101/2021.05.23.21257669; this version posted May 25, 2021. The copyright holder for this preprint (which was not certified by peer review) is the author/funder, who has granted medRxiv a license to display the preprint in perpetuity.

It is made available under a CC-BY-NC-ND 4.0 International license .

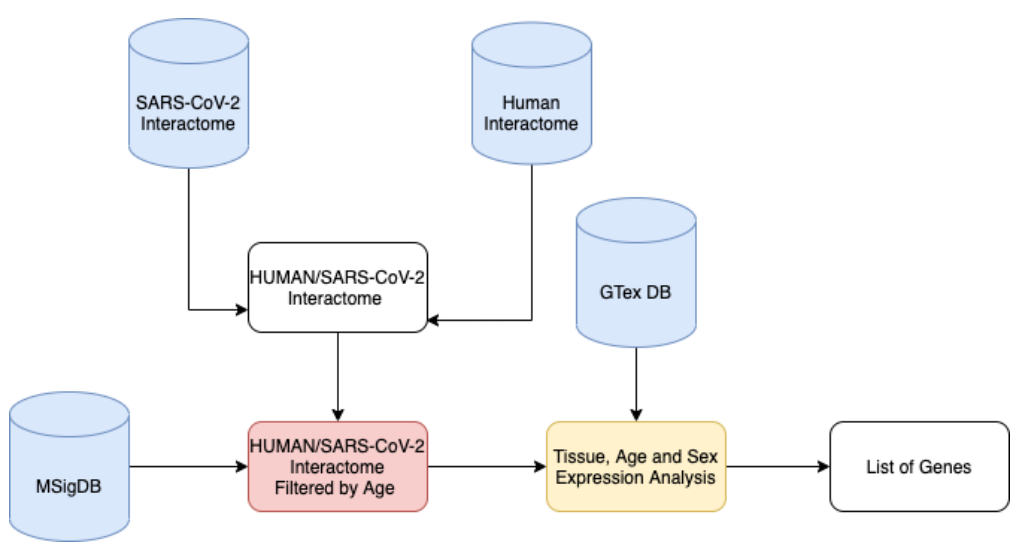

Figure 1: Workflow of the experiment. We downloaded public available interaction data from previous studies. We built the integrated human/SARSCoV-2 interactome. In parallel we downloaded the list of genes annotated with ageing keywords as in MSigDB database. Then, for each SARS-CoV-2 protein we calculated the probability that it contains human interactors annotated with with ageing keyword. We obtained a list of SARS-CoV-2 proteins containing a significant number of interactors related to aging. Then we calculated the intersection of these sets (core interactors) obtaining a list of eight human proteins. For each core interactor, we also considered the expression at tissue level extracting data from GTeX database. We verified that there exist a significant fraction of interacting partners of SARS-CoV-2 that are involved in ageing and that are particularly expressed in lung and in adipose tissue. 
with human age [53]. We gathered data from the GenAge dataset that derived human genes by projecting sequence orthologs in model organisms. We also considered the MSigDB gene set collections, which summarized gene information associated with ageing collected from 70 different studies. We selected datasets reporting experiments from homo sapiens since orthologs' projection may produce not reliable results for ageing as described in [36].

We used the Search Tool for the Retrieval of Interacting Genes/Proteins database (STRING) [54] that is a freely available repository storing both physical and functional association among proteins. Users may search the database through a web interface by specifying a protein identifier or inserting the primary sequence. We queried the database using the identifiers of the nodes of each subnetwork. We used medium confidence as the minimum confidence score for each interaction and all for the sources of interactions. We searched the GTeX Portal [55] using the previously described list of gens. We obtained the expression of those genes in a heat map that shows expression across all GTEx tissues. Gene Ontology analysis was performed by using Gene Ontology web portal [56] while Reactome Database was used for identifying related pathways [57].

Bioinformatic Analysis We selected all known SARS-CoV-2 interacting partners. Then, we measured the intersection between this list of interactors and the ageing-related genes for each viral protein and estimated the probability that this intersection is higher than chance by Fisher's exact test.

We also tested the significance of the difference in the expression of EEF2, NPM1, HMGA1, HMGA2, APEX1, CHEK1, PRKDC, and GPX4 due to age (we considered six different classes), sex, and tissue. We used a Wilcoxon Test for testing difference in the expression among classes (since the expression of genes is not gaussian as reported by a Shapiro test). In addition, the difference among age classes is evaluated using a Kruskal Wallis test.

\section{Results}

\subsection{Network Analysis}

For each viral protein we select the human interactors. The analysis revealed that only ten viral proteins (M, NSP2, NSP4, NSP6, NSP11, NSP13, Orf3a, Orf7a, Orf8, and Orf9c) have interactors with a significant overlap with respect to ageing related proteins, as summarised in Table 1. Then, we considered those that are enriched for ageing in a 
medRxiv preprint doi: https://doi.org/10.1101/2021.05.23.21257669; this version posted May 25, 2021. The copyright holder for this preprint (which was not certified by peer review) is the author/funder, who has granted medRxiv a license to display the preprint in perpetuity.

It is made available under a CC-BY-NC-ND 4.0 International license.

Table 1: P-Values of the enrichment. For each protein we report the significance of the enrichment. A p-value lower than 0.01 means that the interactors are significantly related to ageing. (NS stands for not significant)

\begin{tabular}{|c|c||c|c|}
\hline Viral Protein & P-Value & Viral Protein & P-Value \\
\hline Spike & NS & E & NS \\
\hline M & $\mathbf{6 . 8 4 E - 0 2 ~}$ & N & NS \\
\hline NSP1 & NS & NSP2 & $\mathbf{0 . 0 0 1 8 2}$ \\
\hline NSP3 & NS & NSP4 & $\mathbf{8 . 3 2 E - 0 3 ~}$ \\
\hline NSP5 & NS & NSP6 & $\mathbf{0 . 0 0 2 6 7 6}$ \\
\hline NSP7 & NS & NSP8 & $\mathbf{0 . 0 0 3 4 2 1}$ \\
\hline NSP9 & NS & NSP10 & NS \\
\hline NSP11 & $\mathbf{0 . 0 0 0 1 8 6 2}$ & NSP12 & NS \\
\hline NSP13 & $\mathbf{0 . 0 0 2 5 7 3}$ & NSP14 & NS \\
\hline NSP15 & NS & NSP16 & NS \\
\hline Orf3a & $\mathbf{5 . 0 6 E - 0 3 ~}$ & Orf3b & NS \\
\hline Orf6 & NS & Orf7a & $\mathbf{0 . 0 0 0 1 7 9 1}$ \\
\hline Orf7b & NS & Orf8 & $\mathbf{0 . 0 0 0 6 9 1 3}$ \\
\hline Orf9b & NS & Orf9c & $\mathbf{1 . 5 0 E - 0 2 ~}$ \\
\hline Orf10 & NS & & \\
\hline
\end{tabular}

significant way. Finally, we intersected all these sets and we obtain a core set of eight proteins: EEF2, NPM1, HMGA1, HMGA2, APEX1, CHEK1, PRKDC and GPX2 (core interactors hereafter) as reported in Figure 2 (see supplementary for the list of interactors for each viral protein, integrated with the topological characteristics of the induced subnetwork in the human interactome).

The Gene Ontology analysis reveals that the whole network is enriched with the following terms: (GO:0090402) oncogene-induced cell senescence, (GO:0035986 ) senescence-associated heterochromatin focus assembly, (GO:2000774) positive regulation of cellular senescence, (GO:2000773) negative regulation of cellular senescence, (GO:2000772) regulation of cellular senescence. The analysis of Reactome DB reveals that the subnetwork is associated with following pathways: Formation of Senescence Associated Heterochromatins Foci (HSA2559584), Host interactions of HIV factors.

\subsection{Expression Analysis}

We searched the GTeX database for the expression (of core interactors as reported in Figure 2 expressed as TPM (Transcripts Per Million). 


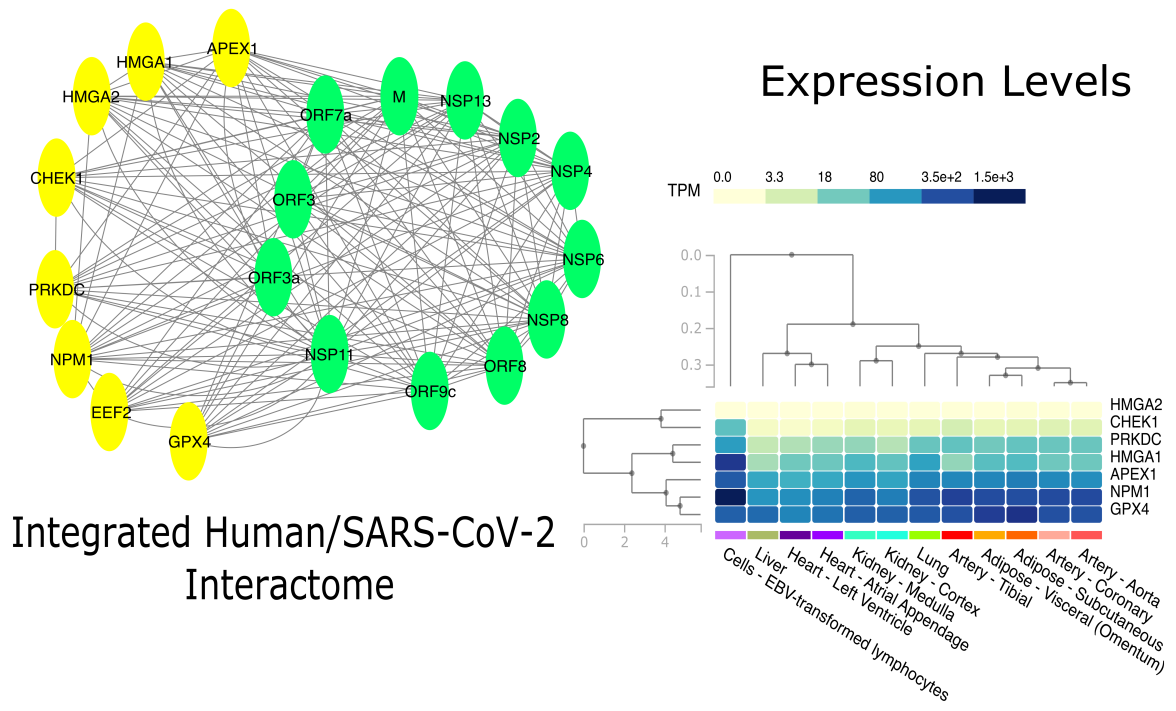

Figure 2: Figure shows tissue level analysis of this work. The Network analysis contributed to find a set of human proteins (yellow nodes) related to aging that interact with many SARS-CoV-2 proteins (green nodes). The analysis of the expression of the related genes at tissue level revealed that all these genes are expressed in lung, as well as in other human tissues. Expression level are presented as TPMS.

We found that all the interactors are expressed in lung as well as in other human tissues (supplementary materials contain the expression levels of these genes in different human tissues). In order to assess the different outcomes between male and female we focused on lung tissue and we compared the expression of these core interactors in male and female as reported in 3. Since data are not normally distributed, (as given by Shapiro Test), we measured the difference of expression in male/female class. We evidenced a significant difference tested by using a Wilcoxon Test for NPM1 and HMGA1 genes that are significantly downregulated in males, without considering age as reported in Figure [? ].

We also explored the trend of the core interactors focusing on lung tissue and six different classes of age (20-29, 30-39, 40-49, 50-59, 60-69, 70-79). We found that there is a significant difference considering age groups for HMGA1, APEX, CHEK1, EEF2, and NPM1 ( $\mathrm{p} \leq 0.05$ as evidenced by a Kruskal Wallis test). Figure 4 report this trend. 
medRxiv preprint doi: https://doi.org/10.1101/2021.05.23.21257669; this version posted May 25, 2021. The copyright holder for this preprint (which was not certified by peer review) is the author/funder, who has granted medRxiv a license to display the preprint in perpetuity.

It is made available under a CC-BY-NC-ND 4.0 International license.
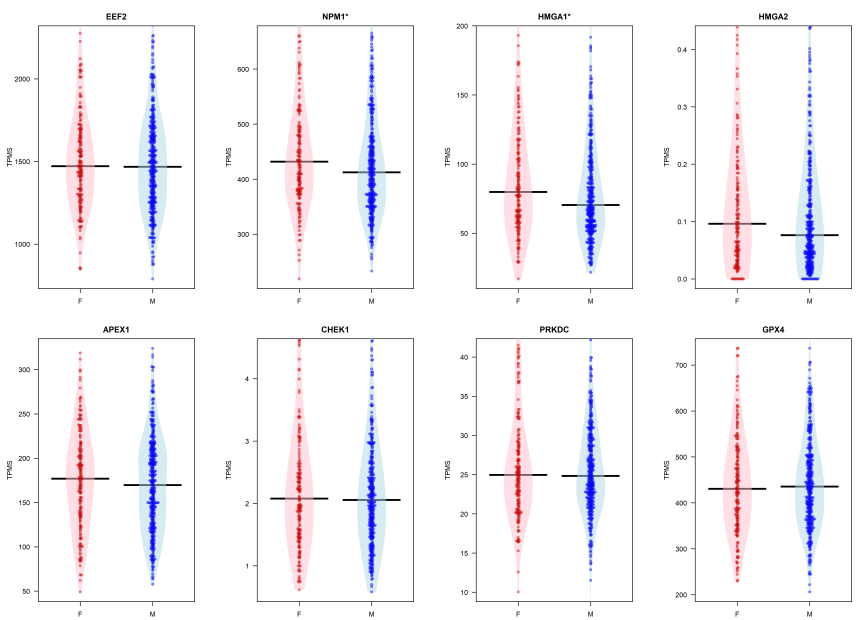

Figure 3: Figure reports box plot of the expression of the eight core genes grouped by sex in the lung tissue. The evidences a significant difference tested by using a Wilcoxon Test for NPM1 and HMGA1 genes.
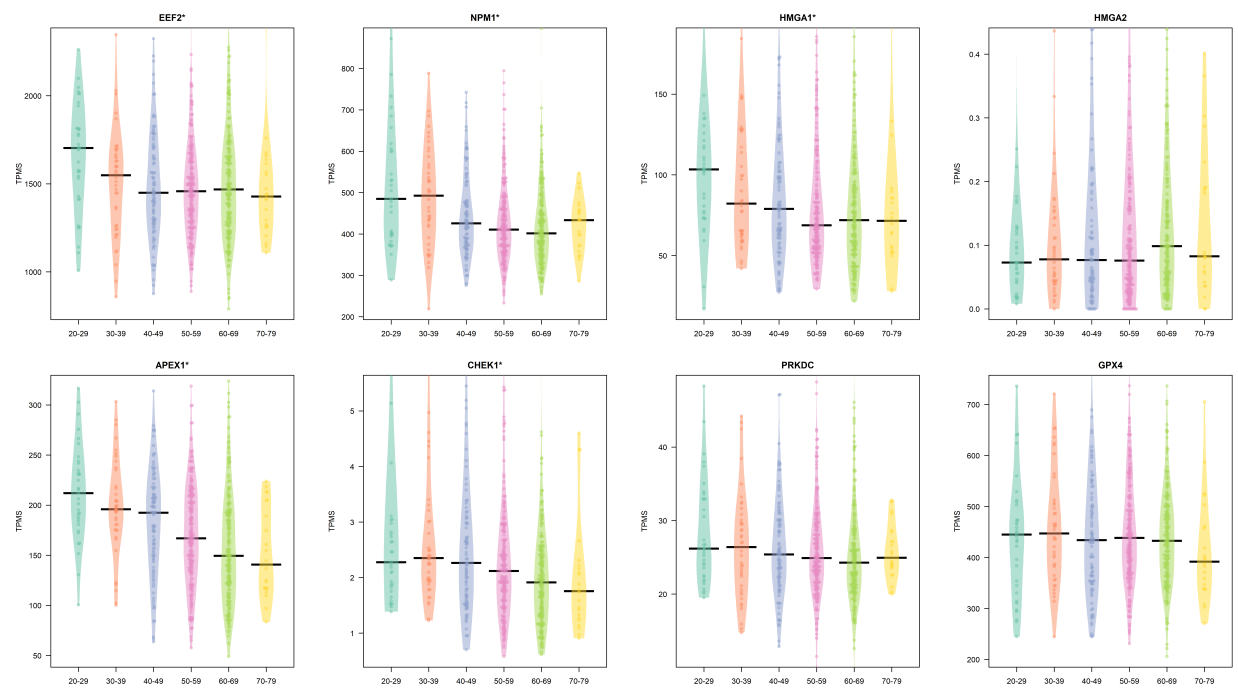

Figure 4: Figure reports the difference of the expression of the core genes in lung tissue in different age classes. A $*$ on top of the plot means a significant difference ( $\mathrm{p} \leq 0.05$ as evidenced by a Kruskal Wallis test) 
We then subdivided these groups considering sex. We found that that females present only the age dependent modulation of APEX1, while in males all the previous genes are modulated considering age groups as reported in Figures 6 (females) and 5 (males) ( $\mathrm{p} \leq 0.05$ as evidenced by a Kruskal Wallis test).

\section{Discussion}

As introduced before, deaths from COVID-19 occur predominantly among older adults. COVID-19 also appears to be more lethal for men rather than women. This characteristic has been found in China, as well as in Europe and in the United States of America, [58].

Starting from this observation, we tried to explain the molecular basis of this phenomenon. Next, we recall that ageing is a heterogeneous process that presents differences among individuals. In particular, age-related changes impact many organs producing possible multiorgan failures even showing many inter-individual differences. Beyond these differences, we tried to explain how the age-related changes at the molecular level can be relevant to COVID-19 pathology.

To achieve this goal, we integrated interactomics and expression data related to COVID-19, age and sex. We started from SARS-CoV-2 interactors, and we isolated age-related from those. Then we considered the expression value of these genes, and we further investigated the trend of changes of these genes in age and sex groups. In summary, we identified a set of statistically significant interactors for the aging process: EEF2, NPM1, HMGA1, HMGA2, APEX1, CHEK1, PRKDC, and GPX4. As reported in Figure 8 we found some interesting changes of these genes considering tissue, age and sex groups. We also found that NPM1 and HMG1 are downregulated in males (statistically significant regulation); while HMGA2 is slightly downregulated in males (not significantly) (Figure 3).

We also found some statistically relevant changes in age for EEF, NPM1, HMGA1, APEX1, and CHEK1 for males (Figure 5), and for APEX1 in Females (Figure 6).

Our findings are coherent with the observation and the literature related to COVID-19.

As investigated in [59] ageing is characterised by the decline of the immune function. Older adults are not immuno-deficient, but often the immune system's response is not sufficient to be effective again antigens. This effect is particularly evident when they are subject to novel antigens. For example, it is known that both responses to influenza and vaccination are not efficient in the elderly $[60,61]$. Moreover, the elderly accumulate inflammatory mediators in tissues 
medRxiv preprint doi: https://doi.org/10.1101/2021.05.23.21257669; this version posted May 25, 2021. The copyright holder for this preprint (which was not certified by peer review) is the author/funder, who has granted medRxiv a license to display the preprint in perpetuity.

It is made available under a CC-BY-NC-ND 4.0 International license .
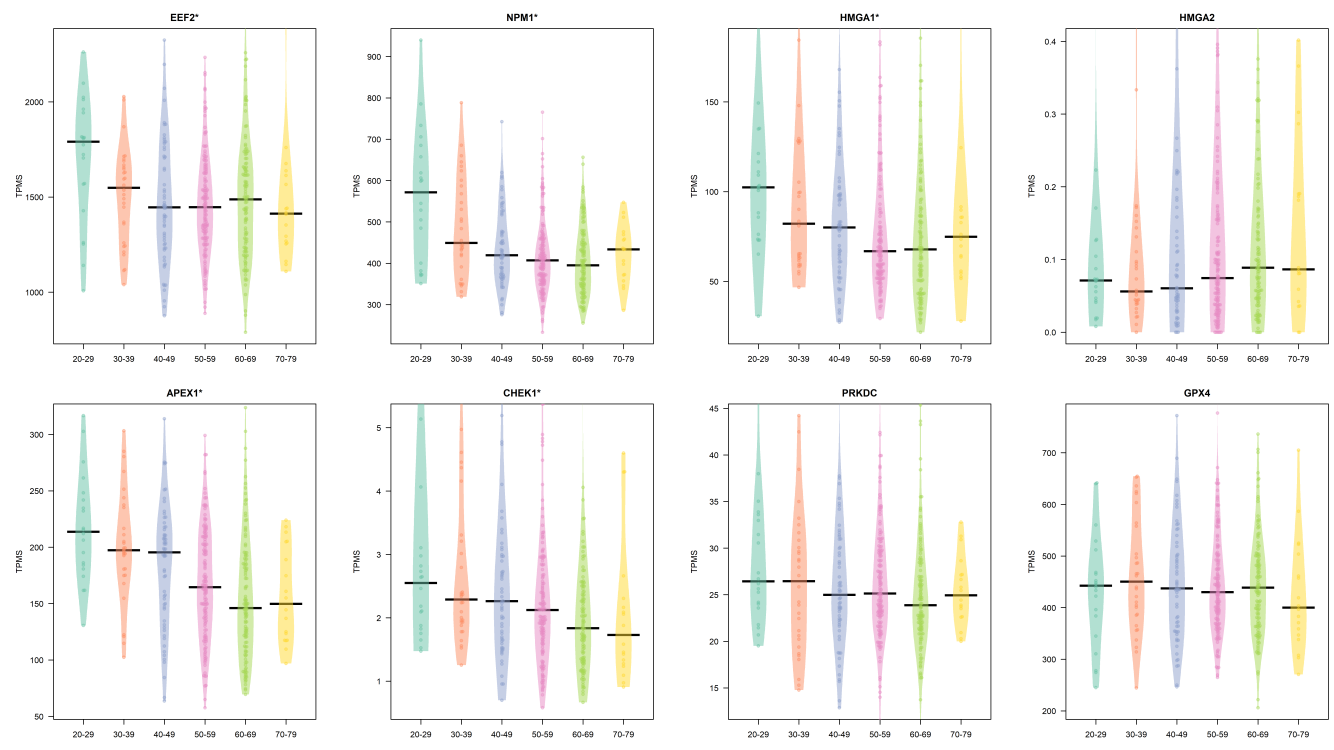

Figure 5: Difference in the expression in lung tissue by age classes in males. Expression is reported as TPM.A * on top reveals a modulation in groups.
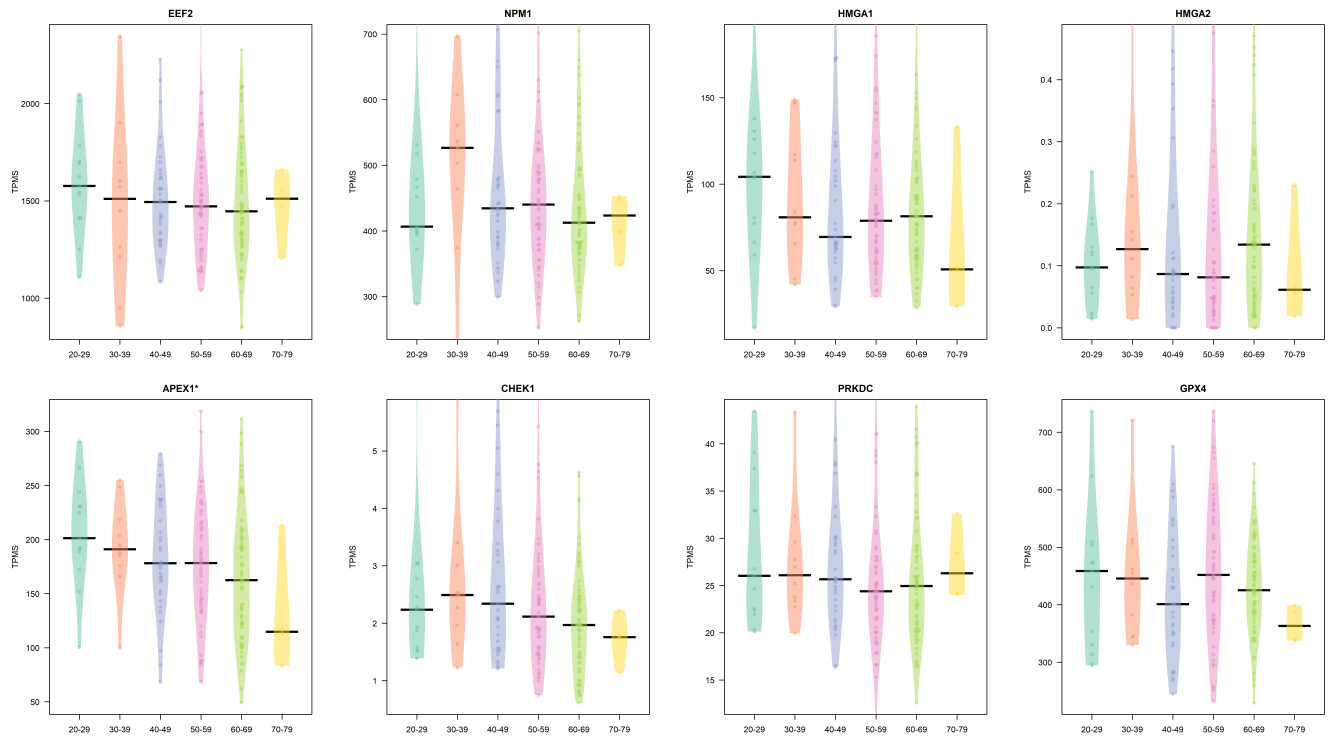

Figure 6: Difference in the expression in lung tissue by age classes in females. Expression is reported as TPM. A * on top reveals a modulation in groups.

Figure 7: Expression of core interactor in males and females grouped by age class. 
(inflammageing process), that may occur by the accumulation of DNA lesions that, in turn, triggers the increased production of inflammatory mediators [62]. In parallel, the link between COVID-19 and the suppression of the immune system has been observed in [63]. Authors found that many proteins related to the immune response were modulated, causing the possible suppression of such a system.

HMGA1 and HMGA2 genes encode four proteins (HMGA1a, HMGA1b, HMGA1c, and HMGA2) belonging to the High-mobility group A (HMGA) protein family [64]. All the proteins bind AT-rich regions in DNA and modulate gene expression by acting as transcription factors. Literature reports that HMGA1 has critical roles in tumorigenesis and the progression of various cancers. However, the role of HMGA1 in COVID-19 has not explored in the past. We now demonstrate that HMGA1 is a SARS-CoV-2 interactor. It is differently regulated in males and the elderly, so these differences in expression are associated with poor prognosis in the found classes (elderly and males). It has been shown that HMGA1 induces inflammatory pathways early in many cancers and pathways involved in stem cells, cell cycle progression, and its dysregulation causes aberration in pathways of stem cells, cellular development, and hematopoiesis [65]. Our results provide insight into HMGA1 function during COVID-19 pathogenesis that could serve as therapeutic targets in human people with aberrant HMGA1 expression.

Similarly to HMGA1, the Nucleophosmin (NPM1) is also downregulated in males. NPM1 is related to DNA and cell cycle control such as ribosome biogenesis, protein chaperoning, centrosome duplication, histone assembly, and cell proliferation [66, 67]. Previous studies investigated the age incidence of acute myeloid leukaemia with mutated nucleophosmin (NPM1) [68, 69], while there are no studies related to these mutations and other diseases. In [? ] the impact of NPM1 modification in older patients has been investigated for AML, suggesting a worse prognosis for older patients due to NPM1 changes. Therefore, this work may stimulate further studies in such a direction. The interaction between NPM1 and the nucleocapsid protein of the previous SARS-CoV is known to affect the viral particle assembly [70, 71, 72]. The role of NPM1 and of Histone H2AX targeted by other viral proteins has also been reported in other viruses such as Epstein-Barr and $\mathrm{KSHV}$ as a common strategy to manipulate translation and to promote virus latency [73, 74].

Moreover, for older men, the scenario is complicated by the downregulation of EEF2, APEX1 and CHEK1.

The dysregulation of EEF2 may cause the accumulation of DNA damage or, on the protein levels accumulating errors in the down- 
stream cascade of mechanisms [75]. The role of EEF2 in severe cases of COVID-19 has also been elucidated in [63]. Thus our study provides another evidence. Moreover, this protein is targeted together with the Eukaryotic translation initiation factor 2 subunit 1 (EIF2S1) by Orf3a, Orf8, NSP2, NSP6, NSP11, NSP13, indicating a possible role of the virus to promote viral translation over cellular translation [76]. In [77] the synergistic downregulation of both APEX1 and NPM1 has been clearly observed in oligodendrocyte cells in relation to ageing. (APEX1) plays an essential role in the cellular response to oxidative stress. APEX1 has a major role in DNA repair and in redox regulation of transcription factors [69]. CHEK1 is targeted together with CDK1 by many SARS-CoV-2 interactors (NSP2, NSP4, NSP11, NSP13) and with CDKN2A (Orf3, NSP13), suggesting an additive effect on the disruption of pathways of apoptosis mediated by TP53 [78] yet dis-regulated by both senescence and ageing. [79].

Differently, for females, we found only the age-dependent modulation of APEX1, suggesting that females may have less factor risk than males. This is coherent with the observation that COVID-19 has a lower CFR rate in females.

In parallel, in supplementary material, we report that that core interactors are also significantly overexpressed in adipose tissue, therefore suggesting a second factor of co-morbidity. Changes in adipose tissue promote a chronic state of low-grade systemic inflammation on a phenotypic level, thus increasing the risk of age-associated diseases $[35,80]$. We here report that core interactors are expressed in adipose tissue, suggesting a possible role that should be further investigated.

\section{Conclusion}

We applied a bioinformatic analysis to perform a qualitative analysis of mechanisms of infection by SARS-CoV-2 in elderly people.

Several studies have shown in the past the modifications of genes and proteins that occur in elderly people. Other studies have partially elucidated the mechanism of infections and the dysregulated pathways in COVID-19 patients.

We detected a statistically significant overlap between SARS-CoV2 interacting proteins and those related to ageing, suggesting a potentially different response in elderly people. Our analysis evidenced that virus infection particularly affects ageing molecular mechanisms centred around proteins EEF2, NPM1, HMGA1, HMGA2, APEX1, CHEK1, PRKDC, and GPX4. We also found that these genes are expressed in lung. Finally we found that there is a significant difference in the expression considering both age and sex. These results will 
medRxiv preprint doi: https://doi.org/10.1101/2021.05.23.21257669; this version posted May 25, 2021. The copyright holder for this preprint (which was not certified by peer review) is the author/funder, who has granted medRxiv a license to display the preprint in perpetuity.

It is made available under a CC-BY-NC-ND 4.0 International license.

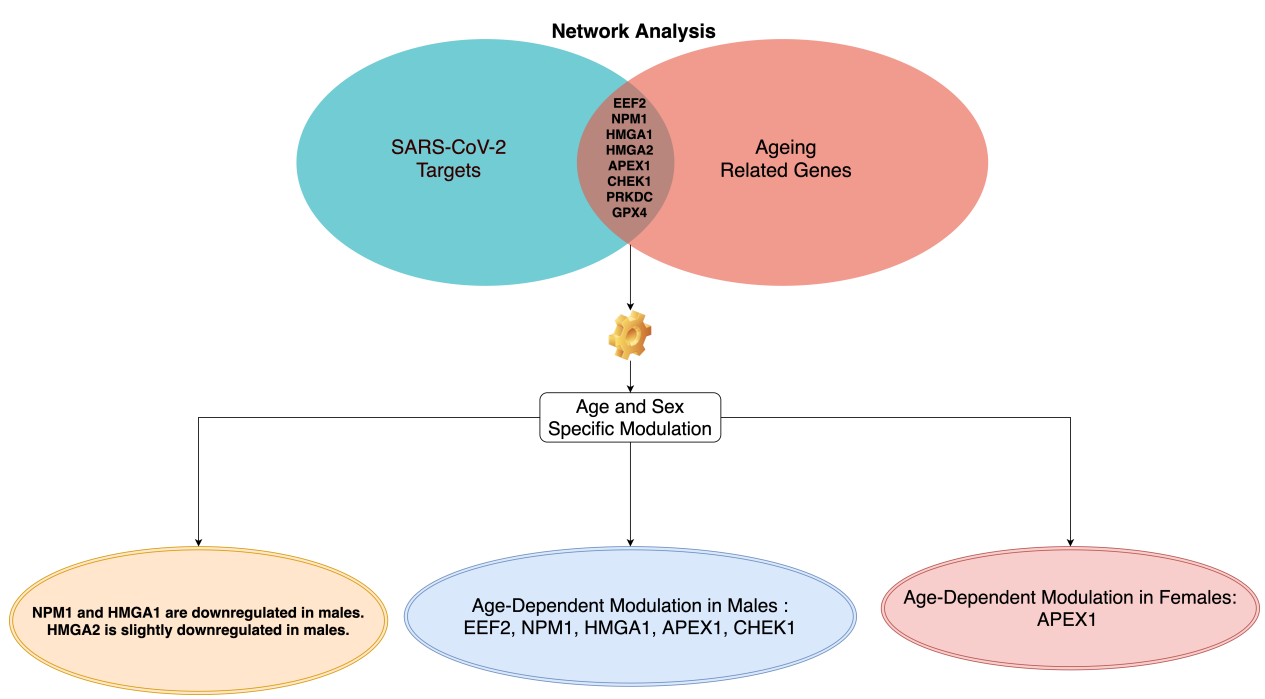

Figure 8: Figure summarises main results of the work. Network analysis found that there exists eight proteins related to ageing that are also all targeted by ten SARS-CoV-2 proteins. The analysis of the expression of their genes revealed that there exist difference on the expression of these genes considering both age and sex.

provide an important molecular basis for understanding the mechanism of infections and will shed light on infection progression. The limitation of this study is that the dataset is correlative, and thus it should be confirmed by in vivo experiments.

\section{Appendix}

\section{Conflict of Interests}

Authors state that they do not have conflict of interest.

\section{Author Contribution}

F.M.G and P.H.G. conceived the main idea of this manuscript. D.M. performed the experimental analysis. Both participated to the experimental phase and on the discussion of the results. E.P. participated to writing of Discussion Section and also validated the clinical aspects of this work. All authors read and approved the manuscript. 


\section{Acknowledgement}

Author thank C.V. Cannistraci for useful suggestions and discussion during the preparation of this work.

\section{$6 \quad$ Key Points}

- A network-based analysis identified some molecular mechanisms that could play a role in the SARS-CoV-2 molecular aetiology and ultimately affect COVID-19 outcome.

- Our analysis evidenced that virus infection particularly affects ageing molecular mechanisms centred around proteins EEF2, NPM1, HMGA1, HMGA2, APEX1, CHEK1, PRKDC, and GPX4.

- We found an age dependent modulation of EEF2, NPM1, HMGA1, APEX1 and CHEK1 in lung tissue of males.

- We found a age dependent modulation of APEX1 in females.

- Our study generated a mechanistic framework aiming at explaining the correlation between COVID-19 incidence in elderly patients and molecular mechanisms of ageing considering differences by age and sex.

\section{Supplementary Materials}

File Supplementary.tex

\section{References}

[1] Talha Burki. Outbreak of coronavirus disease 2019. The Lancet Infectious Diseases, 20(3):292-293, 2020.

[2] Jayanta Kumar Das, Swarup Roy, and Pietro Hiram Guzzi. Analyzing host-viral interactome of sars-cov-2 for identifying vulnerable host proteins during covid-19 pathogenesis. Infection, Genetics and Evolution, page 104921, 2021.

[3] Chaolin Huang, Yeming Wang, Xingwang Li, Lili Ren, Jianping Zhao, Yi Hu, Li Zhang, Guohui Fan, Jiuyang Xu, Xiaoying Gu, et al. Clinical features of patients infected with 2019 novel coronavirus in wuhan, china. The Lancet, 395(10223):497-506, 2020. 
[4] Dawei Wang, Bo Hu, Chang Hu, Fangfang Zhu, Xing Liu, Jing Zhang, Binbin Wang, Hui Xiang, Zhenshun Cheng, Yong Xiong, et al. Clinical characteristics of 138 hospitalized patients with 2019 novel coronavirus-infected pneumonia in wuhan, china. Jama, 323(11):1061-1069, 2020.

[5] Christopher M Petrilli, Simon A Jones, Jie Yang, Harish Rajagopalan, Luke O'Donnell, Yelena Chernyak, Katie A Tobin, Robert J Cerfolio, Fritz Francois, and Leora I Horwitz. Factors associated with hospital admission and critical illness among 5279 people with coronavirus disease 2019 in new york city: prospective cohort study. $b m j, 369,2020$.

[6] Flavia Biamonte, Cirino Botta, Maria Mazzitelli, Salvatore Rotundo, Enrico Maria Trecarichi, Daniela Foti, Carlo Torti, Giuseppe Viglietto, Daniele Torella, and Francesco Costanzo. Combined lymphocyte/monocyte count, d-dimer and iron status predict covid-19 course and outcome in a long-term care facility. Researchsquare, 2020.

[7] Gholam Hossein Meftahi, Zohreh Jangravi, Hedayat Sahraei, and Zahra Bahari. The possible pathophysiology mechanism of cytokine storm in elderly adults with covid-19 infection: the contribution of "inflame-aging". Inflammation Research, pages 1-15, 2020 .

[8] Pietro Hiram Guzzi, Giuseppe Tradigo, and Pierangelo Veltri. Spatio-temporal resource mapping for intensive care units at regional level for covid-19 emergency in italy. International journal of environmental research and public health, 17(10):3344, 2020.

[9] Ensheng Dong, Hongru Du, and Lauren Gardner. An interactive web-based dashboard to track covid-19 in real time. The Lancet infectious diseases, 20(5):533-534, 2020.

[10] Pietro H Guzzi, Giuseppe Tradigo, and Pierangelo Veltri. Regional resource assessment during the covid-19 pandemic in italy: Modeling study. JMIR Medical Informatics, 9(3):e18933, 2021.

[11] Juan David Ramírez, Marina Muñoz, Carolina Hernández, Carolina Flórez, Sergio Gomez, Angelica Rico, Lisseth Pardo, Esther C Barros, and Alberto E Paniz-Mondolfi. Genetic diversity among sars-cov2 strains in south america may impact performance of molecular detection. Pathogens, 9(7):580, 2020. 
[12] Bette Korber, Will M Fischer, Sandrasegaram Gnanakaran, Hyejin Yoon, James Theiler, Werner Abfalterer, Nick Hengartner, Elena E Giorgi, Tanmoy Bhattacharya, Brian Foley, et al. Tracking changes in sars-cov-2 spike: evidence that d614g increases infectivity of the covid-19 virus. Cell, 182(4):812-827, 2020.

[13] Ida Gagliardi, Gemma Patella, Ashour Michael, Raffaele Serra, Michele Provenzano, and Michele Andreucci. Covid-19 and the kidney: From epidemiology to clinical practice. Journal of Clinical Medicine, 9(8):2506, 2020.

[14] Mario Cannataro and Andrew Harrison. Bioinformatics helping to mitigate the impact of covid-19-editorial, 2021.

[15] Daniele Mercatelli, Andrew N Holding, and Federico M Giorgi. Web tools to fight pandemics: the covid-19 experience. Briefings in Bioinformatics, 2020.

[16] Francesco Ortuso, Daniele Mercatelli, Pietro Hiram Guzzi, and Federico Manuel Giorgi. Structural genetics of circulating variants affecting the sars-cov-2 spike/human ace2 complex. Journal of Biomolecular Structure and Dynamics, pages 1-11, 2021.

[17] Johnah C Galicia, Pietro H Guzzi, Federico M Giorgi, and Asma A Khan. Predicting the response of the dental pulp to sarscov2 infection: a transcriptome-wide effect cross-analysis. Genes E Immunity, 21(5):360-363, 2020.

[18] Gabriele Sorci, Bruno Faivre, and Serge Morand. Explaining among-country variation in covid-19 case fatality rate. Scientific reports, 10(1):1-11, 2020.

[19] Wayne C Koff and Michelle A Williams. Covid-19 and immunity in aging populations - a new research agenda. New England Journal of Medicine, 2020.

[20] Gabriella Marcon, Mauro Tettamanti, Giorgia Capacci, Giulia Fontanel, Marco Spanò, Alessandro Nobili, Gianluigi Forloni, and Claudio Franceschi. Covid-19 mortality in lombardy: the vulnerability of the oldest old and the resilience of male centenarians. Aging (Albany NY), 12(15):15186, 2020.

[21] Smriti Mallapaty. The coronavirus is most deadly if you are older and male-new data reveal the risks. Nature, pages 16-17, 2020. 
[22] Tao Li, Lei Lu, Weishuo Zhang, Yu Tao, Liuming Wang, Jing Bao, Bao Liu, and Jun Duan. Clinical characteristics of 312 hospitalized older patients with covid-19 in wuhan, china. Archives of gerontology and geriatrics, 91:104185, 2020.

[23] Carlo Vittorio Cannistraci and Ilaria Capua. Age-adaptive and gender-balanced social interventions might be stepping stones to the next 'new normal'in covid-19 outbreak. Preprints, 2020.

[24] Marianna Milano and Mario Cannataro. Statistical and networkbased analysis of italian covid-19 data: Communities detection and temporal evolution. International journal of environmental research and public health, 17(12):4182, 2020.

[25] Seung-Ji Kang and Sook In Jung. Age-related morbidity and mortality among patients with covid-19. Infection $\&$ chemotherapy, 52(2):154, 2020.

[26] Laura Matrajt, Julia Eaton, Tiffany Leung, and Elizabeth R Brown. Vaccine optimization for covid-19: who to vaccinate first? medRxiv, 2020.

[27] Anna Julia Pietrobon, Franciane Mouradian Emidio Teixeira, and Maria Notomi Sato. I mmunosenescence and inflammaging: Risk factors of severe covid-19 in older people. Frontiers in Immunology, 11, 2020.

[28] Jon Hazeldine and Janet M Lord. Immunesenescence: a predisposing risk factor for the development of covid-19? Frontiers in immunology, 11:2381, 2020.

[29] Claudio Napoli, Isabella Tritto, Gelsomina Mansueto, Enrico Coscioni, and Giuseppe Ambrosio. Immunosenescence exacerbates the covid-19. Archives of Gerontology and Geriatrics, 2020.

[30] Giacomo Grasselli, Antonio Pesenti, and Maurizio Cecconi. Critical care utilization for the covid-19 outbreak in lombardy, italy: early experience and forecast during an emergency response. Jama, 323(16):1545-1546, 2020.

[31] Annemarie B Docherty, Ewen M Harrison, Christopher A Green, Hayley E Hardwick, Riinu Pius, Lisa Norman, Karl A Holden, Jonathan M Read, Frank Dondelinger, Gail Carson, et al. Features of 20133 uk patients in hospital with covid-19 using the isaric who clinical characterisation protocol: prospective observational cohort study. $b m j, 369,2020$. 
[32] Eleonora Cianflone, Michele Torella, Flavia Biamonte, Antonella De Angelis, Konrad Urbanek, Francesco S Costanzo, Marcello Rota, Georgina M Ellison-Hughes, and Daniele Torella. Targeting cardiac stem cell senescence to treat cardiac aging and disease. Cells, 9(6):1558, 2020.

[33] Arne N. Akbar and Derek W. Gilroy. Aging immunity may exacerbate covid-19. Science, 369(6501):256-257, 2020.

[34] C. Franceschi, P. Garagnani, P. Parini, C. Giuliani, and A. Santoro. Inflammaging: a new immune-metabolic viewpoint for agerelated diseases. Nat Rev Endocrinol, 14(10):576-590, 102018.

[35] A. Santoro, G. Guidarelli, R. Ostan, E. Giampieri, C. Fabbri, C. Bertarelli, C. Nicoletti, F. Kadi, L. C. P. G. M. de Groot, E. Feskens, A. Berendsen, A. Brzozowska, O. Januszko, K. Kozlowska, S. Fairweather-Tait, A. Jennings, N. Meunier, E. Caumon, A. Napoli, D. Mercatelli, G. Battista, M. Capri, C. Franceschi, and A. Bazzocchi. Gender-specific association of body composition with inflammatory and adipose-related markers in healthy elderly Europeans from the NU-AGE study. Eur Radiol, 29(9):4968-4979, Sep 2019.

[36] Fazle E Faisal and Tijana Milenković. Dynamic networks reveal key players in aging. Bioinformatics, 30(12):1721-1729, 2014.

[37] Davide Bolignano, Francesco Mattace-Raso, Eric JG Sijbrands, and Carmine Zoccali. The aging kidney revisited: a systematic review. Ageing research reviews, 14:65-80, 2014.

[38] Csaba Kerepesi, Bálint Daróczy, Ádám Sturm, Tibor Vellai, and András Benczúr. Prediction and characterization of human ageing-related proteins by using machine learning. Scientific reports, 8(1):4094, 2018.

[39] L Abenavoli, C Luigiano, PH Guzzi, N Milic, C Morace, L Stelitano, P Consolo, S Miraglia, S Fagoonee, C Virgilio, et al. Serum adipokine levels in overweight patients and their relationship with non-alcoholic fatty liver disease. Panminerva medica, 56(2):189-193, 2014.

[40] Pietro H Guzzi and Mario Cannataro. $\mu$-cs: An extension of the tm4 platform to manage affymetrix binary data. BMC bioinformatics, 11(1):315, 2010. 
[41] Robi Tacutu, Thomas Craig, Arie Budovsky, Daniel Wuttke, Gilad Lehmann, Dmitri Taranukha, Joana Costa, Vadim E Fraifeld, and Joao Pedro De Magalhaes. Human ageing genomic resources: integrated databases and tools for the biology and genetics of ageing. Nucleic acids research, 41(D1):D1027-D1033, 2012.

[42] Pietro Hiram Guzzi and Tijana Milenković. Survey of local and global biological network alignment: the need to reconcile the two sides of the same coin. Briefings in bioinformatics, 19(3):472-481, 2018.

[43] Giovanni Nassa, Roberta Tarallo, Pietro H Guzzi, Lorenzo Ferraro, Francesca Cirillo, Maria Ravo, Ernesto Nola, Marc Baumann, Tuula A Nyman, Mario Cannataro, et al. Comparative analysis of nuclear estrogen receptor alpha and beta interactomes in breast cancer cells. Molecular BioSystems, 7(3):667-676, 2011.

[44] Mikhail V Blagosklonny. From causes of aging to death from covid-19. Aging (Albany NY), 12(11):10004, 2020.

[45] Upasana Bhattacharyya and BK Thelma. Age-related gene expression alterations by sars-cov-2 infection contribute to poor prognosis in elderly. Journal of Genetics, 99(1):1-9, 2020.

[46] Jayanta Kumar Das, Giuseppe Tradigo, Pierangelo Veltri, Pietro H Guzzi, and Swarup Roy. Data science in unveiling covid-19 pathogenesis and diagnosis: evolutionary origin to drug repurposing. Briefings in Bioinformatics, 22(2):855-872, 2021.

[47] Young-Rae Cho, Marco Mina, Yanxin Lu, Nayoung Kwon, and Pietro H Guzzi. M-finder: Uncovering functionally associated proteins from interactome data integrated with go annotations. Proteome science, 11(S1):S3, 2013.

[48] M Bernabeu-Wittel, JE Ternero-Vega, P Díaz-Jiménez, C CondeGuzmán, MD Nieto-Martín, L Moreno-Gaviño, J DelgadoCuesta, M Rincón-Gómez, L Giménez-Miranda, MD NavarroAmuedo, et al. Death risk stratification in elderly patients with covid-19. a comparative cohort study in nursing homes outbreaks. Archives of gerontology and geriatrics, 91:104240, 2020.

[49] C. Robba, D. Battaglini, P. Pelosi, and P. R. M. Rocco. Multiple organ dysfunction in SARS-CoV-2: MODS-CoV-2. Expert Rev Respir Med, 14(9):865-868, 092020. 
[50] Pietro H Guzzi, Daniele Mercatelli, Carmine Ceraolo, and Federico M Giorgi. Master regulator analysis of the sars-cov2/human interactome. Journal of clinical medicine, 9(4):982, 2020 .

[51] David E Gordon, Gwendolyn M Jang, Mehdi Bouhaddou, Jiewei $\mathrm{Xu}$, Kirsten Obernier, Kris M White, Matthew J O'Meara, Veronica V Rezelj, Jeffrey Z Guo, Danielle L Swaney, et al. A sars-cov-2 protein interaction map reveals targets for drug repurposing. Nature, pages 1-13, 2020.

[52] Arthur Liberzon, Aravind Subramanian, Reid Pinchback, Helga Thorvaldsdóttir, Pablo Tamayo, and Jill P Mesirov. Molecular signatures database (msigdb) 3.0. Bioinformatics, 27(12):17391740, 2011.

[53] Kaiwen Jia, Chunmei Cui, Yuanxu Gao, Yuan Zhou, and Qinghua Cui. An analysis of aging-related genes derived from the genotype-tissue expression project (gtex). Cell death discovery, 4(1):1-14, 2018.

[54] Damian Szklarczyk, Annika L Gable, Katerina C Nastou, David Lyon, Rebecca Kirsch, Sampo Pyysalo, Nadezhda T Doncheva, Marc Legeay, Tao Fang, Peer Bork, et al. The string database in 2021: customizable protein-protein networks, and functional characterization of user-uploaded gene/measurement sets. $\mathrm{Nu}$ cleic Acids Research, page gkaa1074, 2020.

[55] John Lonsdale, Jeffrey Thomas, Mike Salvatore, Rebecca Phillips, Edmund Lo, Saboor Shad, Richard Hasz, Gary Walters, Fernando Garcia, Nancy Young, et al. The genotype-tissue expression (gtex) project. Nature genetics, 45(6):580-585, 2013.

[56] Gene Ontology Consortium. The gene ontology (go) database and informatics resource. Nucleic acids research, 32(suppl_1):D258D261, 2004.

[57] Antonio Fabregat, Konstantinos Sidiropoulos, Phani Garapati, Marc Gillespie, Kerstin Hausmann, Robin Haw, Bijay Jassal, Steven Jupe, Florian Korninger, Sheldon McKay, et al. The reactome pathway knowledgebase. Nucleic acids research, 44(D1):D481-D487, 2016.

[58] M Cristina Polidori, Helmut Sies, Luigi Ferrucci, and Thomas Benzing. Covid-19 mortality as a fingerprint of biological age. Ageing Research Reviews, page 101308, 2021. 
[59] MR Encarnacion, BM Beata, and D Kenneth. Cause, consequences, and reversal of immune system aging. J Clin Investig, $123: 958-65,2013$.

[60] William W Thompson, David K Shay, Eric Weintraub, Lynnette Brammer, Nancy Cox, Larry J Anderson, and Keiji Fukuda. Mortality associated with influenza and respiratory syncytial virus in the united states. Jama, 289(2):179-186, 2003.

[61] Thomas A Reichert, Lone Simonsen, Ashutosh Sharma, Scott A Pardo, David S Fedson, and Mark A Miller. Influenza and the winter increase in mortality in the united states, 1959-1999. American journal of epidemiology, 160(5):492-502, 2004.

[62] Claudio Franceschi and Judith Campisi. Chronic inflammation (inflammaging) and its potential contribution to age-associated diseases. Journals of Gerontology Series A: Biomedical Sciences and Medical Sciences, 69(Suppl_1):S4-S9, 2014.

[63] Wenmin Tian, Nan Zhang, Ronghua Jin, Yingmei Feng, Siyuan Wang, Shuaixin Gao, Ruqin Gao, Guizhen Wu, Di Tian, Wenjie Tan, et al. Immune suppression in the early stage of covid-19 disease. Nature communications, 11(1):1-8, 2020.

[64] Isabelle Cleynen and Wim JM Van de Ven. The hmga proteins: a myriad of functions. International journal of oncology, 32(2):289305, 2008.

[65] Andrew Schuldenfrei, Amy Belton, Jeanne Kowalski, C Conover Talbot, Francescopaolo Di Cello, Weijie Poh, Hua-Ling Tsai, Sandeep N Shah, Tait H Huso, David L Huso, et al. Hmga1 drives stem cell, inflammatory pathway, and cell cycle progression genes during lymphoid tumorigenesis. BMC genomics, 12(1):1$17,2011$.

[66] David J López, Ander de Blas, Mikel Hurtado, Mikel GarcíaAlija, Jon Mentxaka, Igor de la Arada, María A Urbaneja, Marián Alonso-Mariño, and Sonia Bañuelos. Nucleophosmin interaction with ape1: Insights into dna repair regulation. DNA repair, 88:102809, 2020.

[67] Gianluca Storci, Maria Giulia Bacalini, Francesca Bonifazi, Paolo Garagnani, Sabrina De Carolis, Stefano Salvioli, Fabiola Olivieri, and Massimiliano Bonafè. Ribosomal dna instability: An evolutionary conserved fuel for inflammaging. Ageing Research Reviews, 58:101018, 2020. 
[68] C Thiede, E Creutzig, D Reinhardt, G Ehninger, and U Creutzig. Different types of npm1 mutations in children and adults: evidence for an effect of patient age on the prevalence of the tctgtandem duplication in npm1-exon 12. Leukemia, 21(2):366-367, 2007.

[69] Helena Johansson and Stina Simonsson. Core transcription factors, oct4, sox2 and nanog, individually form complexes with nucleophosmin (npm1) to control embryonic stem (es) cell fate determination. Aging (Albany NY), 2(11):815, 2010.

[70] Yingchun Zeng, Linbai Ye, Shengli Zhu, Hong Zheng, Peng Zhao, Weijia Cai, Liya Su, Yinglong She, and Zhenghui Wu. The nucleocapsid protein of sars-associated coronavirus inhibits b23 phosphorylation. Biochemical and biophysical research communications, 369(2):287-291, 2008.

[71] Lei Sun, Pan Li, Xiaohui Ju, Jian Rao, Wenze Huang, Shaojun Zhang, Tuanlin Xiong, Kui Xu, Xiaolin Zhou, Lili Ren, Qiang Ding, Jianwei Wang, and Qiangfeng Cliff Zhang. In vivo structural characterization of the whole sars-cov-2 rna genome identifies host cell target proteins vulnerable to re-purposed drugs. bioRxiv, 2020.

[72] Marcel Levi. Covid-19 coagulopathy vs disseminated intravascular coagulation. Blood advances, 4(12):2850-2850, 2020.

[73] Myung-Soo Kang and Elliott Kieff. Epstein-barr virus latent genes. Experimental \& molecular medicine, 47(1):e131-e131, 2015 .

[74] Timsy Uppal, Sagarika Banerjee, Zhiguo Sun, Subhash C Verma, and Erle S Robertson. Kshv lana - the master regulator of kshv latency. Viruses, 6(12):4961-4998, 2014.

[75] Brenna C Beckelman, Wenzhong Yang, Nicole P Kasica, Helena R Zimmermann, Xueyan Zhou, C Dirk Keene, Alexey G Ryazanov, Tao Ma, et al. Genetic reduction of eef2 kinase alleviates pathophysiology in alzheimer's disease model mice. The Journal of clinical investigation, 129(2):820-833, 2019.

[76] Zane A Jaafar and Jeffrey S Kieft. Viral rna structure-based strategies to manipulate translation. Nature Reviews Microbiology, 17(2):110-123, 2019. 
[77] Alerie G de la Fuente, Rayner ML Queiroz, Tanay Ghosh, Christopher E McMurran, Juan F Cubillos, Dwight E Bergles, Denise C Fitzgerald, Clare A Jones, Kathryn S Lilley, Colin P Glover, et al. Changes in the oligodendrocyte progenitor cell proteome with ageing. Molecular \& Cellular Proteomics, 2020.

[78] Yong Xiong, Yuan Liu, Liu Cao, Dehe Wang, Ming Guo, Ao Jiang, Dong Guo, Wenjia Hu, Jiayi Yang, Zhidong Tang, et al. Transcriptomic characteristics of bronchoalveolar lavage fluid and peripheral blood mononuclear cells in covid-19 patients. Emerging microbes $\mathcal{E}$ infections, 9(1):761-770, 2020.

[79] A Rufini, P Tucci, I Celardo, and G Melino. Senescence and aging: the critical roles of p53. Oncogene, 32(43):5129-5143, 2013.

[80] Peter Mancuso and Benjamin Bouchard. The impact of aging on adipose function and adipokine synthesis. Frontiers in endocrinology, 10:137, 2019. 